\section{The Scanning Confocal Electron Microscope}

Nestor J. Zaluzec

Argonne National Laboratory

Zaluzec@aaem.amc.anl.gov

Imaging of sub-micron, sub-surface features of thick optically dense materials at high resolution has always been a difficult and/or time consuming task in materials research. For the most part this role has been relegated to technologically complex and expensive instrumentation having highly penetrating radiation, such as the synchrotron-based Scanning Transmission X-ray Microscope (STXM) or involves the careful preparation of thin cross-section slices for study using the Transmission/Scanning Transmission Electron Microscope (TEM/STEM).

The Scanning Confocal Electron Microscope (SCEM), is an electron-optical implementation of the Scanning Confocal Optical Microscope (SCOM) which permits the observation and characterization of sub-surface structures of thick, optically opaque materials (Zaluzec 2003). The device merges the concept of confocal imaging with the ease of use of the Scanning Electron Microscope (SEM), the penetration ability of the scanning, transmission and $\mathrm{x}$-ray microscopes, and achieves unprecedented resolutions in optically dense materials. It provides both large fields of view as well as a nanometer scale spatial resolution. The SCEM functions as much as 100 times faster than the STXM, yet can be constructed for only a fraction of the cost.

The SCEM differs from the SCOM in the sense that all optical microscopes, scanning enabled or not, employ visible (or near visible) light as both their illumination and detection signals. This restricts the SCOM and its family of related instruments to the study of materials which are optically transparent for the wavelengths employed. To-date, this has resulted in SCOMs being adopted as an imaging tool mainly in the life science community. The use of SCOM in nonoptically-transparent materials in the materials science community has as a consequence been relegated mainly to imaging only the surfaces of solids, a task which is often accomplished using the SEM because of it's generally superior resolution.

We have over the last few years, modified the ANL AAEM (Zaluzec-1991), and then developed and refined the technology in order to implement the principles of confocal imaging into the electron optical column as illustrated in figure 1 . The basic components of the SCEM consist of: a high brightness, high voltage (50-300 kV). field emission electron source and aperture system (1), at least one illumination lens(2), the sample and stage (3), at least one imaging lens (4), the detector aperture and detector (5), illumination scanning system (6) and de-scanning system (7). Ancillary detectors can be also added to the instrument to augment the SCEMs characterization capabilities using such techniques as $x$-ray energy dispersive spectroscopy (XEDS), however these are not required to implement the SCEM mode, they are, however, valued peripherals.

In brief, in a confocal microscope, the source (1), specimen (3) and detector (5) are conjugate image points in a optical system. Imaging is accomplished by adjusting the illumination and detection lenses to bring the source, sample, and detector into focal conjunction. As the illumination is scanned across the specimen, a position resolved de-scanned signal is developed from the intensity measured on the conjugate detector (Minsky 1961). This signal is subsequently used to modulate a computationally synchronized display producing an image. The experienced microscopist will note

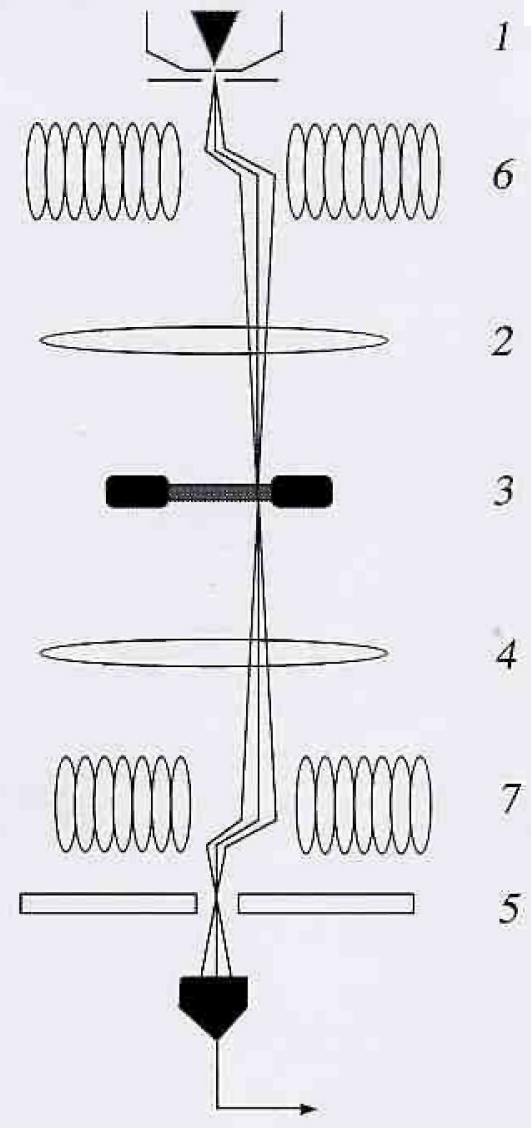

Figure 1: Schematic Diagram of the principle components of the ANL SCEM

the similiarity to STEM mode in figure 1 , this is not a coincidence, as STEM is a subset of the more general SCEM mode, the difference being in the configuration and implementations used for the post specimen lenses.

The use of confocal mode with its appropriate aperturing, lens setting, scanning and de-scanning systems allows the optimization of signal collection and most importantly the removal of spurious or undesirable scattering which blurs and/or obscures image details. In implementing the confocal mode, one substantially extends the range and thickness of a material from which usable images can be obtained, when compared to conventional electron microscopy methods. This is elucidated further by the examples in figures $2-4$ (Note: in the images that follow, we have adjusted both the contrast and brightness to achieve suitable ranges for printing as per the MSA image processing guidelines).

The principle functionality of SCEM at this time, resides in its ability to image subsurface features in extremely thick materials. In figure 2 , we demonstrate this capability by first comparing low magnification images of an integrated circuit bonding pad $(-80 \times$ 80 microns) on a self supporting $\sim 10$ micron thick substrate. Figure $2 \mathrm{~A}$ presents an optical image of the bond pad, while figure $2 \mathrm{~B}$ the corresponding SEM image of the same area. Both of these micrographs illustrate the problem of using visible light or secondary electrons. Although surface details are well resolved, sub-surface structures are obscured. Figure $2 \mathrm{C}$ is a low magnification $300 \mathrm{kV}$ TEM image of the same bond pad illustrating the blurring which results from multiple inelastic scattering sources which obscure the image detail and rendering the image virtually useless. Figure 2D, presents the corresponding SCEM image, dramatically Illustrating how the confocal mode minimizes the blurring effect of the scat- 

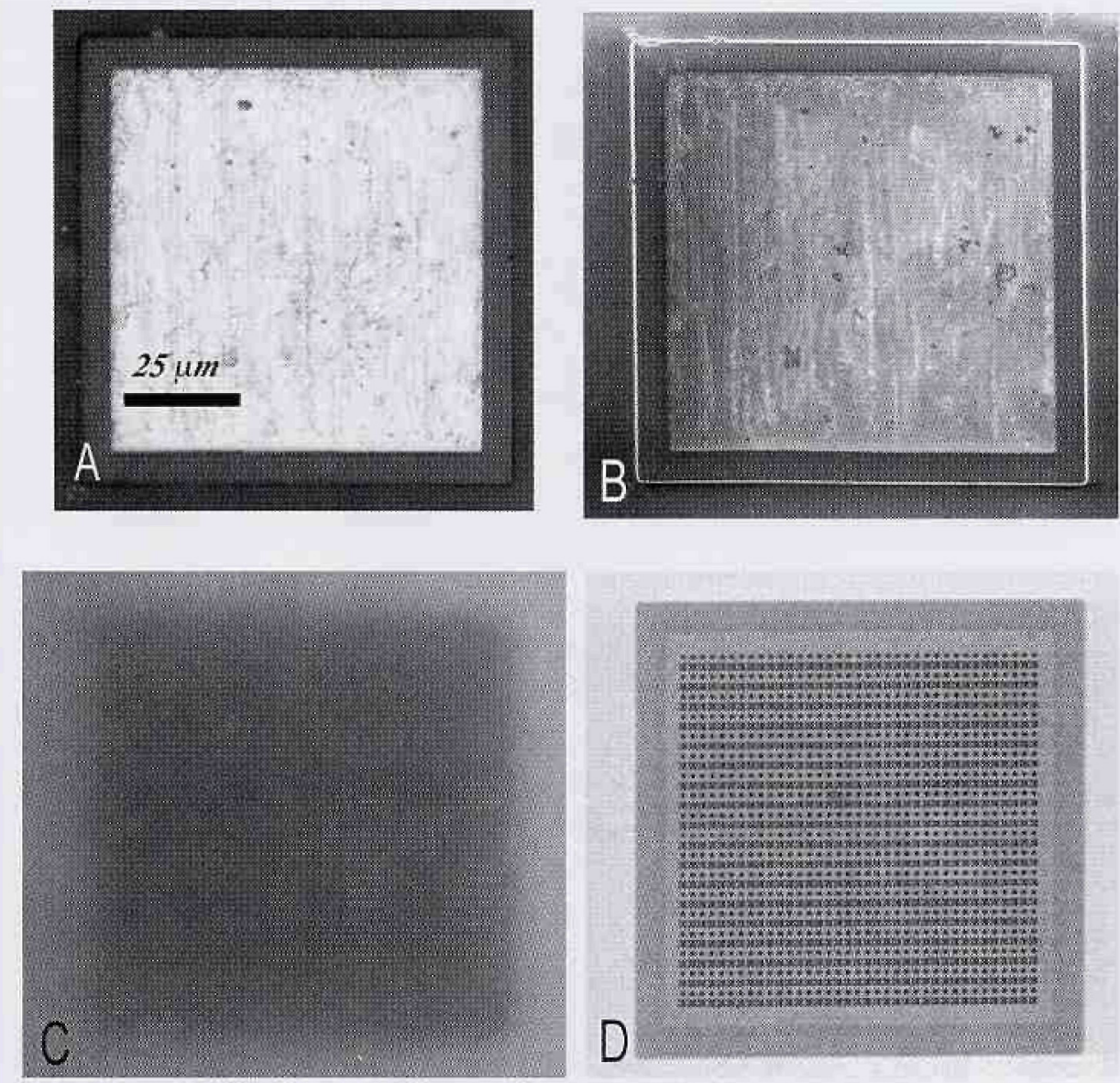

Figure 2: Comparison of Optical (A), SEM (B). TEM (C) and SCEM (D) images of the same bond pad in a semiconductor device.
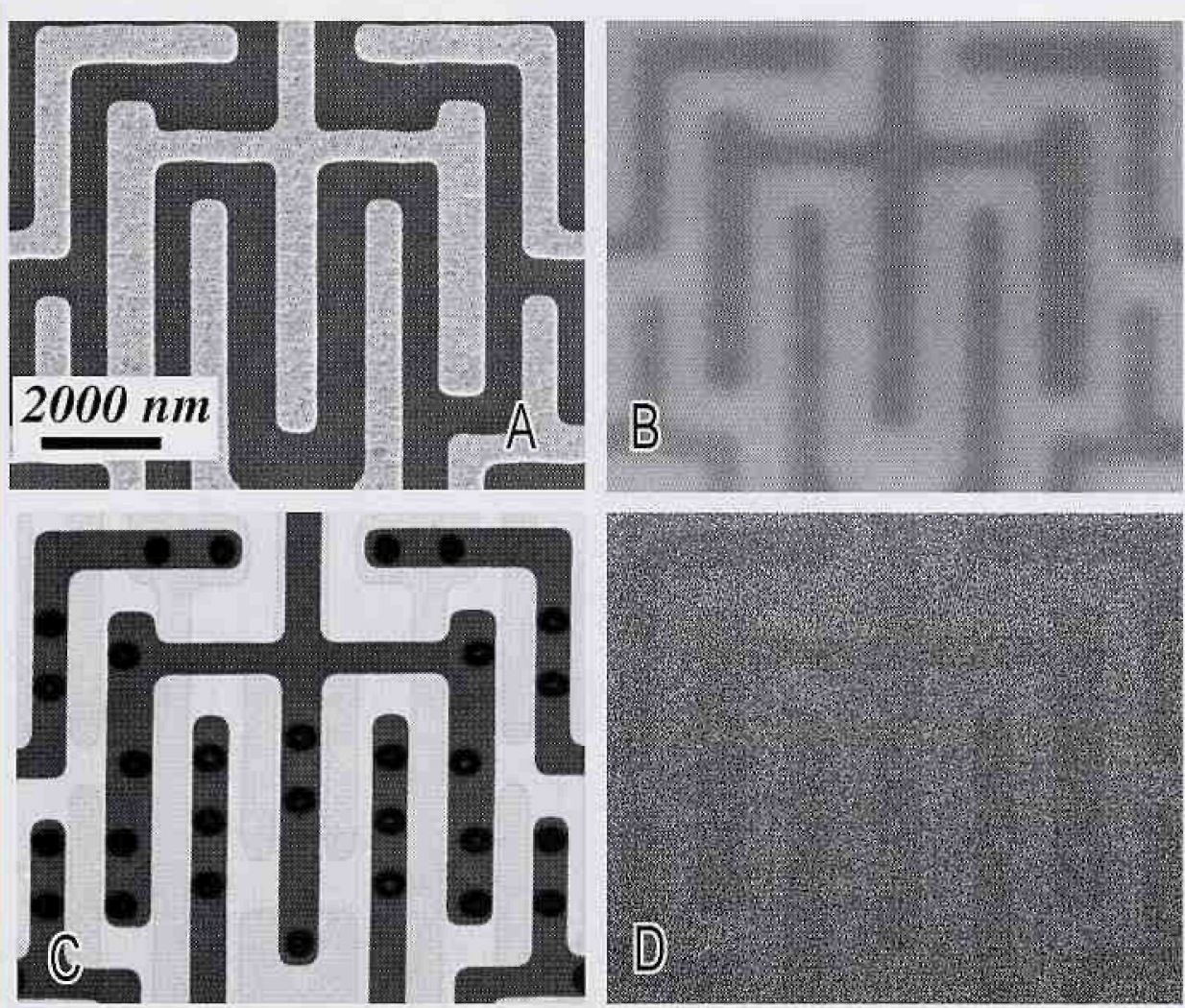

Figure 3: Comparison of SEM (A), TEM (B), SCEM (C) and EF-STEM (D) images of an integrated circuit. tering and permits the direct observation of sub-surface features.

Figure 3 presents a higher resolution example taken from a thick ( $\sim 4$ micron) multilayered microelectronic device and compares the SEM (3A), TEM (3B) and the SCEM (3C) modes. As in the previous case, we see that the SEM produces a very detailed image of the surface of the device, but little else. The TEM, hints at the subsurface structure (the dark vias along the conduction paths) but it yields virtually no usable information about the internal structure of sample due to the extreme blurring caused by multiple scattering processes. The SCEM image (3C), however, provides images of a wealth of sub-surface features, including the interconnect layers and the vias between layers all of which are undetected by both SEM and TEM. For comparison, figure 3D presents an energy filtered (elastic) STEM image of the same area, which is nearly devoid of signal as the mean energy loss of this area exceeds $2 \mathrm{keV}$, the corresponding energy filtered TEM image will be similar.

Because of the lack of useable information derived from TEM imaging of thick specimens such as these, the STXM has frequently been the instrument of choice for imaging of buried structures in integrated circuits. Having a spatial resolution of $\sim 130 \mathrm{~nm}$ (Levine et al. 2000 ) it requires minimal specimen preparation when compared to cross-sectional TEM and has been used successfully for the study of submicron structures relevant to the microelectronics community.

We next compare in figure 4 , high resolution images of an electromigration void formed in an integrated circuit having undergone accelerated life testing. The void in the conduction path was formed in buried Titanium-Nitride (TiN) coated Aluminum (AI) wires $(-1000 \mathrm{~nm}$ wide) that are interconnected by Tungsten (W) vias all of which were located $\sim 5$ microns beneath the surface of a $\sim 10$ micron thick specimen. Figure $4 \mathrm{~A}$, shows the SEM image of the area of interest, in which one can only observe locating fiducial marks produced by a Focussed Ion Beam (FIB ) instrument. In figure $4 \mathrm{~B}$, we present the STXM image of the same area in which one clearly resolves the two level metallization stack, the interconnect lines, the vias, the FIB marks and the extended void that formed in the lower wire. Figure $4 \mathrm{C}$, presents the corresponding SCEM image. It is pertinent to point out that the images shown in figure 4 were all acquired from the same specimen, without any additional preparation. Furthermore, while the image quality of the 


\section{Piecing Together Analytical Solutions}
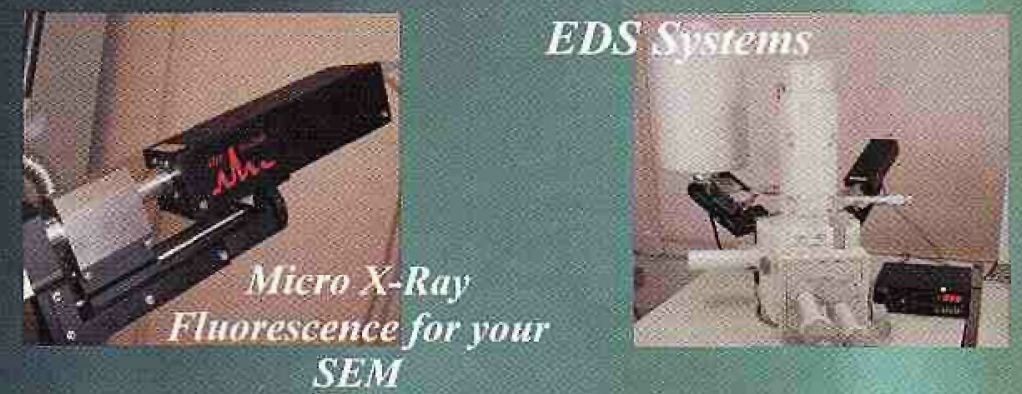
SEM



Advanced EDDS Qumatitation

LXRF Systems, Inc: 15715 Brookford Dr. Houston, TX, 77059 USA
Whether it is Digital Image Analysis, X-ray Mapping or Quantitative EDS you are looking for, LXRF has your solution. Designed to meet the analytical needs of you, the user, simple yet powerfol tools provide a price to performance ratio that is unparalleled.

- Flexible, full customization throughout the software

- Comprehensive, adaptive spectrum processing and the highest level of quantitative analysis

- Powerful, automated, and complete statistical digital image analysis that includes Feature Analysis on phases or particles

O Highly interactive, showcase, fullspectrum $x$-ray mapping that includes fractionated phase or chemical composition statistical analysis

Remember: As evidence of our commitment to our customers, IXRF will provide Free software upgrades for as long as you own your system. Our software is improved and upgraded largely based on customer suggestions.

But this should be old news. Over the past decade IXRF has fundamentally changed the industry. As another example of our dedication to innovtion, we are offering, with an industry first, the addition of small-spot XRF analysis to the world of Scanning Electron Mícroscopy. Experience elemental sensitivities and detection limits that are 10100 times greater than electron beam excitation. Imagine looking at a sample and seeing 5 elemental peaks. NOW, imagine "discovering" an additional 25 peaks!

We pride ourselves in customer service. Call us at (281) 286-6485 to talk to any of our stati, or ask one of our hundreds of customers-after all- they ${ }^{3}$ re our best sales people! 

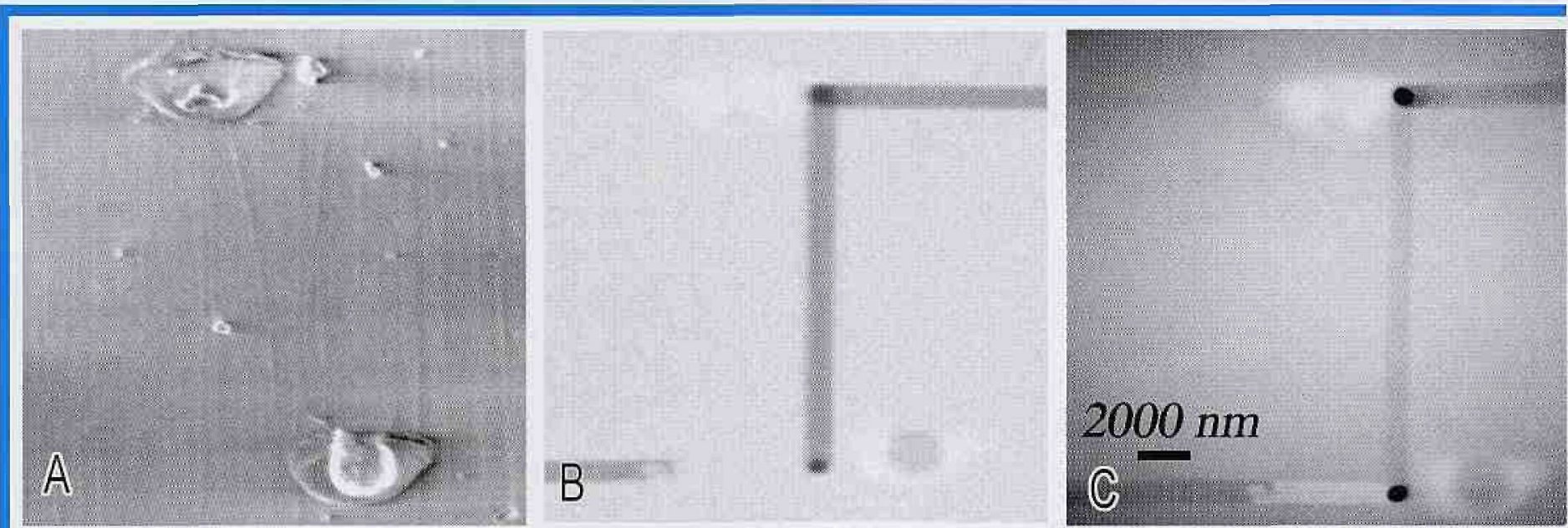

Figure 4: Comparison of SEM (A), STXM (B), and SCEM (C) images of an electromigration void.

STXM and SCEM images are comparable, it should be appreciated that the STXM image was acquired using a Synchrotron Beam Line at the ANL Advanced Photon Source. The acquisition time of the 301 $x 301$ pixel STXM image was $\sim 60$ minutes, and necessitated the coordinated efforts of synchrotron beam line operators, technicians, and the individual scientists involved in the measurements, all of which was necessarily conducted at a large multi-user, multi-million dollar national user facility. In contrast, the SCEM image was measured by a single person working alone, in the relative convenience of a standard laboratory space, the data was acquired at higher definition (pixel density) and at acquisition times typical of a conventional high resolution SEM imaging.

Ultimately, the resolution of the SCEM is limited by probe scattering in transmission mode. Figure 5 plots the experimental resolution of the prototype instrument in a multilayer semiconductor device as a function of total thickness. We have operated the instrument from $100-300 \mathrm{kV}$ and as one would expect resolution in thick specimens is best at the higher accelerating voltage. Currently, the use of SCEM for depth sectioning is limited by the effective aperture angles of the illumination and detection lens systems in the ANL instrument, work in this area is in on-going and should improve with developments in aberration correction such as those proposed in the TEAM project (Kabius, et al. 2003).

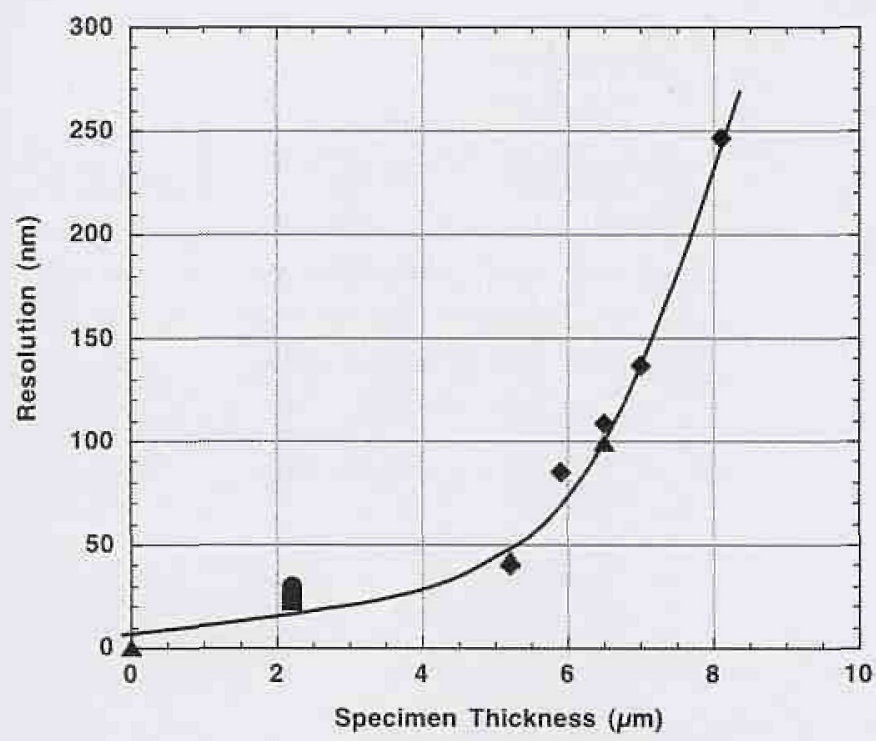

Figure 5: Experimental measurements of resolution as a function of specimen thickness in the ANL SCEM. EO $=300 \mathrm{kV}$.

\section{References:}

Kabius, B., Allen C., Miller D., "Aberration Correction for Analytical In-situ TEM the NTEAM Concept", Proc. of Microscopy \& Microanalysis 2002 Levine, Z., et al. "Tomography of Integrated Circuit Interconnect with an Electromigration Void", J. of Applied Physics. Vol. 87, No. 9, 2000.

Minsky, M., US Patent \# 3,013,467, (1961): Minsky, M., Scanning 10 , pge 128-138 (1988).

Zaluzec, N.J. "The ANLAdvanced Analytical Electron Microscope" in MicroBeam Analysis-1991, San Francisco Press, 1991, Ed. D. Howitt.

Zaluzec, N.J., US Patent \#6,548,810-0 (2003). This work has been supported by the US DoE at ANL under contract \#BES-MS W-31-109-Eng-38.

\section{Nestor Zaluzec Wins Prestigious R\&D 100 Award for Developing the SCEM}

This is 40 th year the technology awards have been given by $R \& D$ Magazine. The awards recognize the " 100 most technologically significant new products" from the entries the magazine receives. The judges look for products that can change people's lives for the better, improve the standard of living for large numbers of people, save lives, promote health, protect the environment or advance our ability to solve vexing problems of importance to the nation and the world.

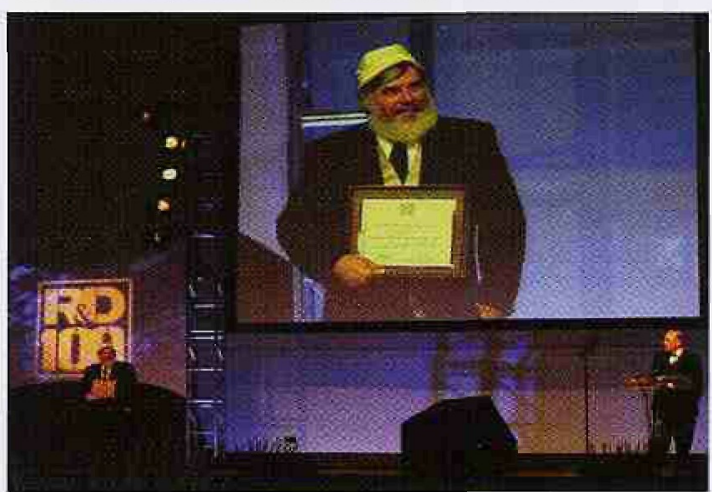

The Scanning Confocal Electron Microscope (SCEM) is an electron optical instrument that merges the principles of confocal imaging by combining the ease of a Scanning Electron Microscope and the penetration ability of both the Scanning Transmission X-ray Microscope and the Transmission/Scanning Transmission Electron Microscope. The SCEM enables imaging of sub-surface structures of thick, optically opaque materials that previously required an $X$-ray microscope. It also enables imaging at large fields of view and at small, billionths-of-a-meter or nanometer-level resolution. The SCEM's main application is in studies of nanomaterials, particularly the next generation of electronic, magnetic and photonic devices. An example of these devices is anything from high tech consumer electronics to the latest in multi-layered high density R\&D integrated circuits.

Excerpted from the Argonne National Laboratory Press Release 


\section{$x^{2}=0$}

THE NEXT GENERATION I MAG ING F I LTER HAS ARRIVED

\section{PRECISION}
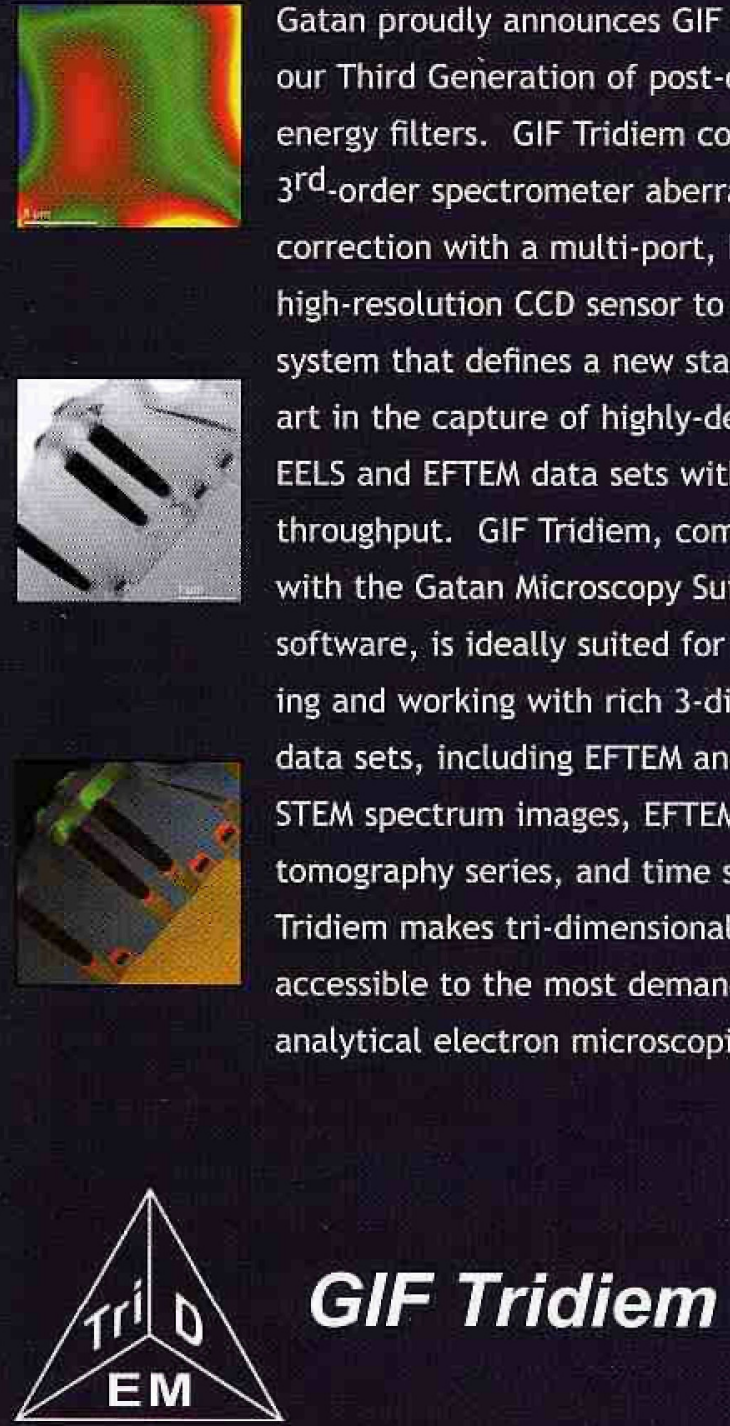

Gatan proudly announces GIF Tridiem, our Third Generation of post-column energy filters. GIF Tridiem combines $3^{\text {rd }}$-order spectrometer aberration correction with a multi-port, high-speed, high-resolution CCD sensor to yield a system that defines a new state-of-theart in the capture of highly-detailed EELS and EFTEM data sets with maximum throughput. GIF Tridiem, combined with the Gatan Microscopy Suite (GMS) software, is ideally suited for generating and working with rich 3 -dimensional data sets, including EFTEM and EELS STEM spectrum images, EFTEM tilt and tomography series, and time series. GIF Tridiem makes tri-dimensional EM readily accessible to the most demanding analytical electron microscopist.

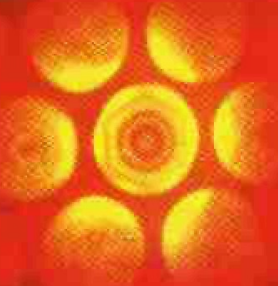

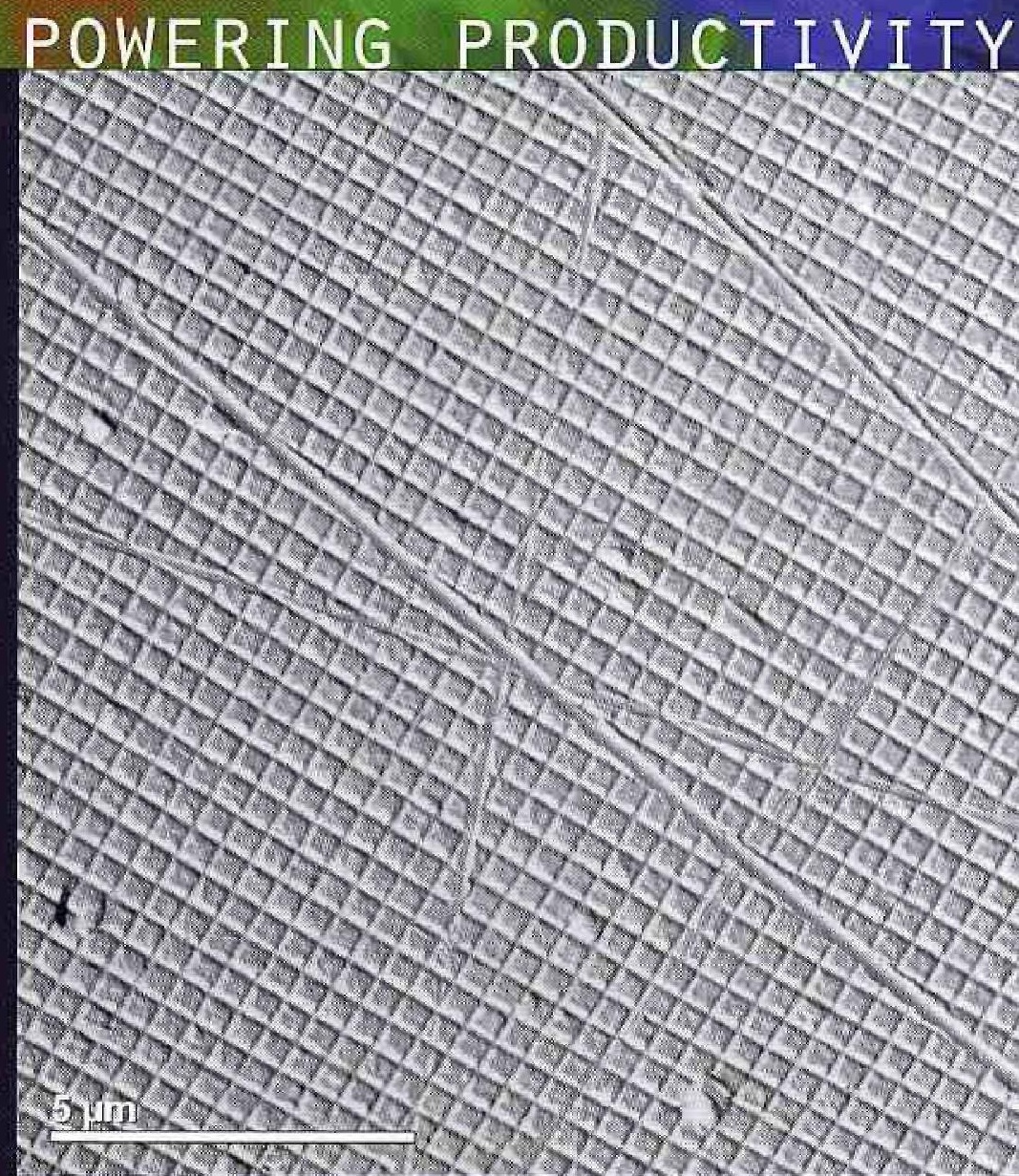

Lefi-hand Images (from top if bottom): (1) Energy deviation across image field corrected thru $3^{\text {rd }}$ order $10<2 \mathrm{eV}$. (2) Unfittered Bright-field TEM image of semicanductor device structure. (3) Color composite of elemental maps of semiconductor device structure.

Above Imagest (lop) Zero-toss filtered CBED pattem of Si ItII taken at $200 \mathrm{keV}$. FOL Z ring represents stallering angle of $78 \mathrm{mR}$. (botiom) Zero-lass image of grating replica showing lavge fiald of view Tor EFTEM Grating pilch is $463 \mathrm{~mm}$.

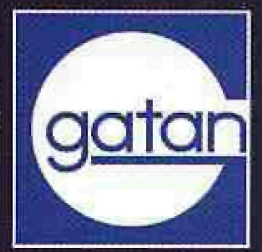

www.gatan.com 